\title{
Bioinformatics analysis of rabbit haemorrhagic disease virus genome
}

\author{
Xiao-ting $\operatorname{Tian}^{\dagger}{ }^{\dagger}$ Bao-yu Li ${ }^{\dagger}$, Liang Zhang, Wen-qiang Jiao and Ji-xing Liu*
}

\begin{abstract}
Background: Rabbit haemorrhagic disease virus (RHDV), as the pathogeny of Rabbit haemorrhagic disease, can cause a highly infectious and often fatal disease only affecting wild and domestic rabbits. Recent researches revealed that it, as one number of the Caliciviridae, has some specialties in its genome, its reproduction and so on.

Results: In this report, we firstly analyzed its genome and two open reading frameworks (ORFs) from this aspect of codon usage bias. Our researches indicated that mutation pressure rather than natural is the most important determinant in RHDV with high codon bias, and the codon usage bias is nearly contrary between ORF1 and ORF2, which is maybe one of factors regulating the expression of VP60 (encoding by ORF1) and VP10 (encoding by ORF2). Furthermore, negative selective constraints on the RHDV whole genome implied that VP10 played an important role in RHDV lifecycle.

Conclusions: We conjectured that VP10 might be beneficial for the replication, release or both of virus by inducing infected cell apoptosis initiate by RHDV. According to the results of the principal component analysis for ORF2 of RSCU, we firstly separated 30 RHDV into two genotypes, and the ENC values indicated ORF1 and ORF2 were independent among the evolution of RHDV.
\end{abstract}

Keywords: Rabbit haemorrhagic disease virus (RHDV), Codon usage, Evolution, Expression

\section{Background}

Synonymous codons are not used randomly [1]. The variation of codon usage among ORFs in different organisms is accounted by mutational pressure and translational selection as two main factors $[2,3]$. Levels and causes of codon usage bias are available to understand viral evolution and the interplay between viruses and the immune response [4]. Thus, many organisms such as bacteria, yeast, Drosophila, and mammals, have been studied in great detail up on codon usage bias and nucleotide composition [5]. However, same researches in viruses, especially in animal viruses, have been less studied. It has been observed that codon usage bias in human RNA viruses is related to mutational pressure, G $+\mathrm{C}$ content, the segmented nature of the genome and

\footnotetext{
* Correspondence: liujixing@hotmail.com

† Contributed equally

State Key Laboratory of Veterinary Etiological Biology, Key Laboratory of Grazing Animal Diseases of Ministry of Agriculture, Key Laboratory of Animal Virology of Ministry of Agriculture, State Key Laboratory of Veterinary Etiological Biology, Lanzhou Veterinary Research Institute, Chinese Academy of Agricultural Sciences, Xujia ping 1, Yanchang bu, Lanzhou, Gansu, Post
} Code 730046 , China

(c) 2011 Tian et al; licensee BioMed Central Ltd. This is an Open Access article distributed under the terms of the Creative Commons Attribution License (http://creativecommons.org/licenses/by/2.0), which permits unrestricted use, distribution, and reproduction in any medium, provided the original work is properly cited. the route of transmission of the virus [6]. For some vertebrate DNA viruses, genome-wide mutational pressure is regarded as the main determinant of codon usage rather than natural selection for specific coding triplets [4]. Analysis of the bovine papillomavirus type 1 (BPV1) late genes has revealed a relationship between codon usage and tRNA availability [7]. In the mammalian papillomaviruses, it has been proposed that differences from the average codon usage frequencies in the host genome strongly influence both viral replication and gene expression [8]. Codon usage may play a key role in regulating latent versus productive infection in EpsteinBarr virus [9]. Recently, it was reported that codon usage is an important driving force in the evolution of astroviruses and small DNA viruses [10,11]. Clearly, studies of synonymous codon usage in viruses can reveal much about the molecular evolution of viruses or individual genes. Such information would be relevant in understanding the regulation of viral gene expression.

Up to now, little codon usage analysis has been performed on Rabbit haemorrhagic disease virus (RHDV), which is the pathogen causing Rabbit haemorrhagic 
disease (RHD), also known as rabbit calicivirus disease (RCD) or viral haemorrhagic disease (VHD), a highly infectious and often fatal disease that affects wild and domestic rabbits. Although the virus infects only rabbits, RHD continues to cause serious problems in different parts of the world. RHDV is a single positive stranded RNA virus without envelope, which contains two open reading frames (ORFs) separately encoding a predicted polyprotein and a minor structural protein named VP10 [12]. After the hydrolysis of self-coding 3C-like cysteinase, the polyprotein was finally hydrolyzed into 8 cleavage products including 7 nonstructural proteins and 1 structural protein named as VP60 [13,14]. Studies on the phylogenetic relationship of RHDVs showed only one serotype had been isolated, and no genotyping for RHDV was reported. It reported that the VP10 was translated with an efficiency of $20 \%$ of the preceding ORF1 [15]. In order to better understand the characteristics of the RHDV genome and to reveal more information about the viral genome, we have analyzed the codon usage and dinucleotide composition. In this report, we sought to address the following issues concerning codon usage in RHDV: (i) the extent and causes of codon bias in RHDV; (ii) A possible genotyping of RHDV; (iii) Codon usage bias as a factor reducing the expression of VP10 and (iiii) the evolution of the ORFs.

\section{Materials and methods}

\subsection{Sequences}

The 30 available complete RNA sequences of RHDV were obtained from GenBank randomly in January 2011. The serial number $(\mathrm{SN})$, collection dates, isolated areas and GenBank accession numbers are listed in Table 1.

\subsection{The relative synonymous codon usage (RSCU) in} RHDV

To investigate the characteristics of synonymous codon usage without the influence of amino acid composition, RSCU values of each codon in a ORF of RHDV were calculated according to previous reports (2 Sharp, Tuohy et al. 1986) as the followed formula:

$$
\mathrm{RSCU}=\frac{g_{i j}}{\sum_{j}^{n_{i}} g_{i j}} n_{i}
$$

Where $g_{i j}$ is the observed number of the $i$ th codon for $j$ th amino acid which has $\mathrm{n}_{\mathrm{i}}$ type of synonymous codons. The codons with RSCU value higher than 1.0 have positive codon usage bias, while codons with value lower than 1.0 has relative negative codon usage bias. As RSCU values of some codons are nearly equal to 1.0 , it means that these codons are chosen equally and randomly.
Table 1 Information of RHDV genomes

\begin{tabular}{|c|c|c|c|c|}
\hline SN & Strain & Isolation & Date & Accession No. \\
\hline 1 & UT-01 & USA:Utah & 2001 & EU003582.1 \\
\hline 2 & NY-01 & USA: New York & 2001 & EU003581.1 \\
\hline 3 & Italy-90 & Italy & 1990 & EU003579.1 \\
\hline 4 & IN-05 & USA: Indiana & 2005 & EU003578.1 \\
\hline 5 & NJ-2009 & China: Nanjing & 2009 & HM623309.1 \\
\hline 6 & lowa2000 & USA: Iowa & 2000 & AF258618.2 \\
\hline 7 & pJG-RHDV-DD06 & Ramsay Island & 2007 & EF363035.1 \\
\hline 8 & Bahrain & Bahrain & 2006 & DQ189077.1 \\
\hline 9 & CD/China & Changchun, China & 2004 & AY523410.1 \\
\hline 10 & RHDV-V351 & Czech & 1996 & U54983.1 \\
\hline 11 & RHDV-Hokkaido & Japan & 2002 & AB300693.2 \\
\hline 12 & RHDV-FRG & Germany & 1991 & NC_001543.1 \\
\hline 13 & Meiningen & Germany & 2007 & EF558577.1 \\
\hline 14 & Jena & Germany & 2007 & EF558576.1 \\
\hline 15 & Hartmannsdorf & Germany & 2007 & EF558586.1 \\
\hline 16 & Rossi & Germany & 2007 & EF558584.1 \\
\hline 17 & Triptis & Germany & 2007 & EF558583.1 \\
\hline 18 & Dachswald & Germany & 2007 & EF558582.1 \\
\hline 19 & Erfurt & Germany & 2007 & EF558581.1 \\
\hline 20 & NZ61 & New Zealand & 2007 & EF558580.1 \\
\hline 21 & NZ54 & New Zealand & 2007 & EF558579.1 \\
\hline 22 & Eisenhuttenstadt & Germany & 2007 & EF558578.1 \\
\hline 23 & Ascot & United Kingdom & 2007 & EF558575.1 \\
\hline 24 & Wika & Germany & 2007 & EF558574.1 \\
\hline 25 & Frankfurt5 & Germany & 2007 & EF558573.1 \\
\hline 26 & Frankfurt12 & Germany & 2007 & EF558572.1 \\
\hline 27 & WHNRH & China & 2005 & DQ280493.1 \\
\hline 28 & BS89 & Italy & 1995 & X87607.1 \\
\hline 29 & RHDV-SD & France & 1993 & Z29514.1 \\
\hline 30 & M67473.1 & Germany & 1991 & M67473.1 \\
\hline
\end{tabular}

2.3 The content of each nucleotides and $\mathrm{G}+\mathrm{C}$ at the synonymous third codon position (GC3s)

The index GC3s means the fraction of the nucleotides $\mathrm{G}+\mathrm{C}$ at the synonymous third codon position, excluding Met, Trp, and the termination codons.

\subsection{The effective number of codons (ENC)}

The ENC, as the best estimator of absolute synonymous codon usage bias [16], was calculated for the quantification of the codon usage bias of each ORF [17]. The predicted values of ENC were calculated as

$$
\mathrm{ENC}=2+s+\frac{29}{s^{2}+\left(1-s^{2}\right)}
$$

where $\mathrm{s}$ represents the given $(\mathrm{G}+\mathrm{C})_{3} \%$ value. The values of ENC can also be obtained by EMBOSS CHIPS program [18]. 


\subsection{Dn and ds of two ORFs}

Analyses were conducted with the Nei-Gojobori model [19], involving 30 nucleotide sequences. All positions containing gaps and missing data were eliminated. The values of $\mathrm{dn}$, ds and $\omega(\mathrm{dn} / \mathrm{ds})$ were calculated in MEGA4.0 [20].

\subsection{Correspondence analysis (COA)}

Multivariate statistical analysis can be used to explore the relationships between variables and samples. In this study, correspondence analysis was used to investigate the major trend in codon usage variation among ORFs. In this study, the complete coding region of each ORF was represented as a 59 dimensional vector, and each dimension corresponds to the RSCU value of one sense codon (excluding Met, Trp, and the termination codons) [21].

\subsection{Correlation analysis}

Correlation analysis was used to identify the relationship between nucleotide composition and synonymous codon usage pattern [22]. This analysis was implemented based on the Spearman's rank correlation analysis way.

All statistical processes were carried out by with statistical software SPSS 17.0 for windows.

\section{Results}

\subsection{Measures of relative synonymous codon usage}

The values of nucleotide contents in complete coding region of all 30 RHDV genomes were analyzed and listed in Table 2 and Table 3. Evidently, $(\mathrm{C}+\mathrm{G}) \%$ content of the ORF1 fluctuated from 50.889 to 51.557 with a mean value of 51.14557 , and $(C+G) \%$ content of the ORF2 were ranged from 35.593 to 40.113 with a mean value of 37.6624 , which were indicating that nucleotides $A$ and $U$ were the major elements of ORF2 against ORF1. Comparing the values of $\mathrm{A}_{3} \%, \mathrm{U}_{3} \%, \mathrm{C}_{3} \%$ and $\mathrm{G}_{3} \%$, it is clear that $\mathrm{C}_{3} \%$ was distinctly high and $\mathrm{A}_{3} \%$ was the lowest of all in ORF1 of RHDV, while $\mathrm{U}_{3} \%$ was distinctly high and $\mathrm{C}_{3} \%$ was the lowest of all in ORF2 of

Table 2 Identified nucleotide contents in complete coding region (length $>250$ bps) in the ORF1 of RHDV (30 isolates) genome

\begin{tabular}{|c|c|c|c|c|c|c|c|c|c|c|c|}
\hline SN & $\mathrm{A} \%$ & $A_{3} \%$ & U\% & $\mathrm{U}_{3} \%$ & $\mathrm{C} \%$ & $\mathrm{C}_{3} \%$ & G\% & $\mathrm{G}_{3} \%$ & $(\mathrm{C}+\mathrm{G}) \%$ & $\left(C_{3}+G_{3}\right) \%$ & ENC \\
\hline 1 & 25.302 & 18.252 & 23.340 & 23.497 & 25.544 & 33.348 & 25.814 & 24.904 & 51.358 & 58.252 & 54.786 \\
\hline 2 & 25.387 & 18.294 & 23.738 & 24.691 & 25.146 & 32.281 & 25.729 & 24.733 & 51.386 & 57.014 & 55.201 \\
\hline 3 & 25.515 & 18.678 & 23.298 & 23.795 & 25.657 & 33.220 & 25.529 & 24.307 & 51.186 & 57.527 & 55.05 \\
\hline 4 & 25.899 & 19.488 & 22.758 & 21.876 & 26.141 & 35.053 & 25.203 & 23.582 & 51.344 & 58.635 & 54.68 \\
\hline 5 & 25.515 & 18.593 & 23.554 & 24.136 & 25.373 & 32.878 & 25.558 & 24.392 & 50.931 & 57.270 & 55.491 \\
\hline 6 & 25.458 & 18.294 & 23.554 & 24.222 & 25.444 & 32.921 & 25.544 & 24.563 & 50.988 & 57.484 & 55.268 \\
\hline 7 & 25.359 & 18.806 & 23.454 & 23.667 & 25.487 & 33.262 & 25.700 & 24.264 & 51.187 & 57.526 & 54.723 \\
\hline 8 & 25.402 & 18.721 & 23.412 & 23.625 & 25.544 & 33.305 & 25.643 & 24.350 & 51.187 & 57.655 & 55.031 \\
\hline 9 & 25.615 & 19.062 & 23.383 & 23.625 & 25.544 & 33.433 & 25.458 & 23.881 & 51.002 & 57.314 & 54.906 \\
\hline 10 & 25.430 & 18.593 & 23.383 & 23.966 & 25.629 & 33.006 & 25.558 & 24.435 & 51.187 & 57.441 & 55.439 \\
\hline 11 & 25.288 & 17.910 & 23.596 & 24.435 & 25.402 & 32.751 & 25.714 & 24.904 & 51.116 & 57.665 & 54.984 \\
\hline 12 & 25.529 & 18.635 & 23.412 & 23.838 & 25.515 & 33.092 & 25.544 & 24.435 & 51.059 & 57.527 & 55.203 \\
\hline 13 & 25.387 & 18.380 & 23.611 & 23.966 & 25.316 & 33.006 & 25.686 & 24.648 & 51.002 & 57.654 & 54.681 \\
\hline 14 & 25.274 & 18.124 & 23.426 & 23.582 & 25.544 & 33.433 & 25.757 & 24.861 & 51.301 & 58.294 & 54.548 \\
\hline 15 & 25.203 & 18.166 & 23.724 & 24.691 & 25.188 & 32.239 & 25.885 & 24.904 & 51.073 & 57.143 & 55.429 \\
\hline 16 & 25.487 & 18.721 & 23.326 & 23.326 & 25.601 & 33.603 & 25.586 & 24.350 & 51.187 & 57.953 & 55.148 \\
\hline 17 & 25.444 & 18.507 & 23.369 & 23.582 & 25.572 & 33.433 & 25.615 & 24.478 & 51.187 & 57.911 & 55.27 \\
\hline 18 & 25.572 & 18.806 & 23.539 & 24.179 & 25.416 & 32.836 & 25.473 & 24.179 & 50.889 & 57.015 & 55.417 \\
\hline 19 & 25.487 & 18.507 & 23.582 & 24.136 & 25.359 & 32.964 & 25.572 & 24.392 & 50.931 & 57.356 & 55.384 \\
\hline 20 & 25.558 & 18.806 & 23.426 & 23.966 & 25.473 & 32.878 & 25.544 & 24.350 & 51.017 & 57.228 & 55.165 \\
\hline 21 & 25.544 & 18.721 & 23.426 & 24.009 & 25.529 & 33.006 & 25.501 & 24.264 & 51.030 & 57.270 & 55.156 \\
\hline 22 & 25.160 & 17.783 & 23.312 & 23.326 & 25.729 & 33.689 & 25.800 & 25.203 & 51.529 & 58.892 & 54.682 \\
\hline 23 & 25.487 & 18.806 & 23.511 & 23.710 & 25.529 & 33.433 & 25.473 & 24.051 & 51.002 & 57.487 & 54.192 \\
\hline 24 & 25.387 & 18.593 & 23.497 & 23.667 & 25.572 & 33.348 & 25.544 & 24.392 & 51.116 & 57.740 & 54.213 \\
\hline 25 & 25.330 & 18.635 & 23.483 & 23.582 & 25.615 & 33.433 & 25.572 & 24.350 & 51.187 & 57.783 & 54.238 \\
\hline 26 & 25.387 & 18.593 & 23.511 & 23.710 & 25.572 & 33.390 & 25.529 & 24.307 & 51.101 & 57.697 & 54.285 \\
\hline 27 & 25.330 & 18.209 & 23.511 & 24.264 & 25.487 & 32.964 & 25.672 & 24.563 & 51.159 & 57.527 & 55.267 \\
\hline 28 & 25.448 & 18.643 & 23.443 & 23.635 & 25.576 & 33.362 & 25.533 & 24.360 & 51.109 & 57.722 & 54.614 \\
\hline 29 & 25.174 & 17.868 & 23.269 & 23.156 & 25.686 & 33.817 & 25.871 & 25.160 & 51.557 & 58.977 & 54.842 \\
\hline 30 & 25.529 & 18.635 & 23.412 & 23.838 & 25.515 & 33.092 & 25.544 & 24.435 & 51.059 & 57.527 & 55.203 \\
\hline
\end{tabular}


Table 3 Identified nucleotide contents in complete coding region (length $>250$ bps) in the ORF2 of RHDV (30 isolates) genome

\begin{tabular}{|c|c|c|c|c|c|c|c|c|c|c|c|}
\hline SN & $A \%$ & $A_{3} \%$ & U\% & $U_{3} \%$ & $\mathrm{C} \%$ & $C_{3} \%$ & G\% & $\mathrm{G}_{3} \%$ & $(C+G) \%$ & $\left(C_{3}+G_{3}\right) \%$ & ENC \\
\hline 1 & 29.944 & 17.797 & 30.791 & 44.068 & 13.842 & 16.102 & 25.424 & 22.034 & 39.266 & 38.136 & 49.377 \\
\hline 2 & 29.944 & 18.644 & 30.226 & 43.220 & 14.407 & 16.949 & 25.424 & 21.186 & 39.831 & 38.135 & 48.182 \\
\hline 3 & 31.356 & 20.339 & 31.638 & 46.610 & 12.994 & 13.559 & 24.011 & 19.492 & 37.005 & 33.051 & 44.567 \\
\hline 4 & 30.508 & 18.644 & 30.791 & 44.915 & 13.842 & 15.254 & 24.859 & 21.186 & 38.701 & 36.440 & 46.686 \\
\hline 5 & 29.944 & 17.797 & 31.921 & 46.610 & 12.712 & 13.559 & 25.424 & 22.034 & 38.136 & 35.593 & 41.215 \\
\hline 6 & 30.226 & 16.949 & 30.226 & 43.220 & 14.407 & 16.949 & 25.141 & 22.881 & 39.548 & 39.830 & 51.964 \\
\hline 7 & 31.356 & 19.492 & 30.791 & 45.763 & 14.124 & 15.254 & 23.729 & 19.492 & 37.853 & 34.764 & 45.757 \\
\hline 8 & 30.226 & 16.949 & 29.661 & 43.220 & 15.254 & 17.797 & 24.859 & 22.034 & 40.113 & 39.831 & 47.242 \\
\hline 9 & 30.508 & 18.644 & 31.356 & 45.763 & 13.277 & 14.407 & 24.859 & 21.186 & 38.136 & 35.593 & 43.017 \\
\hline 10 & 31.356 & 20.339 & 31.638 & 46.610 & 12.994 & 13.559 & 24.011 & 19.492 & 37.005 & 33.051 & 44.576 \\
\hline 11 & 29.782 & 17.518 & 33.898 & 48.175 & 12.107 & 13.139 & 24.213 & 21.168 & 36.320 & 34.307 & 43.088 \\
\hline 12 & 31.638 & 21.186 & 31.073 & 45.763 & 12.994 & 13.559 & 24.294 & 19.492 & 37.288 & 33.051 & 44.997 \\
\hline 13 & 31.073 & 18.644 & 31.638 & 46.610 & 13.277 & 14.407 & 24.011 & 20.339 & 37.288 & 34.746 & 43.213 \\
\hline 14 & 31.638 & 19.492 & 31.921 & 47.458 & 12.994 & 13.559 & 23.446 & 19.492 & 36.440 & 33.051 & 47.214 \\
\hline 15 & 31.921 & 20.339 & 31.921 & 46.610 & 12.712 & 13.559 & 23.446 & 19.492 & 36.158 & 33.051 & 41.964 \\
\hline 16 & 30.226 & 18.644 & 30.508 & 43.220 & 14.124 & 16.949 & 25.141 & 21.186 & 39.265 & 38.135 & 47.603 \\
\hline 17 & 30.508 & 19.492 & 30.508 & 43.220 & 13.559 & 15.254 & 25.424 & 22.034 & 38.983 & 37.288 & 47.615 \\
\hline 18 & 29.096 & 16.102 & 31.356 & 45.763 & 13.277 & 14.407 & 26.271 & 23.729 & 39.548 & 38.136 & 44.343 \\
\hline 19 & 30.226 & 19.492 & 31.073 & 44.915 & 13.559 & 15.254 & 25.141 & 20.339 & 38.700 & 35.593 & 46.768 \\
\hline 20 & 31.638 & 19.492 & 32.768 & 49.153 & 11.864 & 11.017 & 23.729 & 20.339 & 35.593 & 31.356 & 39.771 \\
\hline 21 & 31.638 & 19.492 & 32.768 & 49.153 & 11.864 & 11.017 & 23.729 & 20.339 & 35.593 & 31.356 & 39.771 \\
\hline 22 & 31.073 & 19.492 & 31.356 & 45.763 & 12.994 & 13.559 & 24.576 & 21.186 & 37.570 & 34.745 & 43.282 \\
\hline 23 & 31.356 & 19.492 & 31.921 & 47.458 & 12.994 & 13.559 & 23.729 & 19.492 & 36.723 & 33.051 & 42.633 \\
\hline 24 & 31.638 & 20.339 & 31.921 & 47.458 & 12.994 & 13.559 & 23.446 & 18.644 & 36.440 & 32.203 & 42.157 \\
\hline 25 & 31.638 & 20.339 & 32.203 & 48.305 & 12.712 & 12.712 & 23.446 & 18.644 & 36.185 & 31.356 & 40.006 \\
\hline 26 & 31.638 & 20.339 & 32.203 & 48.305 & 12.712 & 12.712 & 23.446 & 18.644 & 36.185 & 31.356 & 40.006 \\
\hline 27 & 30.226 & 17.797 & 31.073 & 44.915 & 13.559 & 15.254 & 25.141 & 22.034 & 38.700 & 37.288 & 42.799 \\
\hline 28 & 31.356 & 18.644 & 31.356 & 45.763 & 13.559 & 15.254 & 23.729 & 20.339 & 37.288 & 35.593 & 45.413 \\
\hline 29 & 31.638 & 21.186 & 31.638 & 46.610 & 12.712 & 12.712 & 24.011 & 19.492 & 36.723 & 32.204 & 43.618 \\
\hline 30 & 31.638 & 21.186 & 31.073 & 45.763 & 12.994 & 13.559 & 24.294 & 19.492 & 37.288 & 32.721 & 44.997 \\
\hline
\end{tabular}

RHDV. The $\left(\mathrm{C}_{3}+\mathrm{G}_{3}\right) \%$ in ORF1 fluctuated from 57.014 to 58.977 with a mean value of 57.68287 and $\left(\mathrm{C}_{3}+\mathrm{G}_{3}\right) \%$ were range from 31.356 to 39.831 with a mean value of 34.8337. And the ENC values of ORF1 fluctuated from 54.192 to 55.491 with a mean value of 54.95 and ENC values of ORF2 displayed a far-ranging distribution from 39.771 to 51.964 with a mean value of 44.46 . The ENC values of ORF1 were a little high indicating that there is a particular extent of codon preference in ORF1, but the codon usage is relatively randomly selected in ORF2 on the base of ENC values. The details of the overall relative synonymous codon usage (RSCU) values of 59 codons for each ORF in 30 RHDV genomes were listed in Table 4. Most preferentially used codons in ORF1 were C-ended or G-ended codons except Ala, Pro and Ser, however, A-ended or G-ended codons were preferred as the content of ORF2.

In addition, the $\mathrm{dn}, \mathrm{ds}$ and $\omega(\mathrm{dN} / \mathrm{dS})$ values of ORF1 were separately $0.014,0.338$ and 0.041 , and the values of
ORF2 were $0.034,0.103$ and 0.034 , respectively. The $\omega$ values of two ORFs in RHDV genome are generally low, indicating that the RHDV whole genome is subject to relatively strong selective constraints.

\subsection{Correspondence analysis}

COA was used to investigate the major trend in codon usage variation between two ORFs of all 30 RHDV selected for this study. After COA for RHDV Genome, one major trend in the first axis $\left(f_{1}\right)$ which accounted for $42.967 \%$ of the total variation, and another major trend in the second axis $\left(f_{2}\right)$ which accounted for $3.632 \%$ of the total variation. The coordinate of the complete coding region of each ORF was plotted in Figure 1 defining by the first and second principal axes. It is clear that coordinate of each ORF is relatively isolated. Interestingly, we found that relatively isolated spots from ORF2 tend to cluster into two groups: the ordinate value of one group (marked as Group 1) is 
Table 4 Synonymous codon usage of the whole coding sequence in RHDV

\begin{tabular}{|c|c|c|c|c|c|c|c|}
\hline$A A^{a}$ & Codon & $\mathrm{RSCU}$ in ORF1 & $\mathrm{RSCU}$ in ORF2 & $A A^{a}$ & Codon & $\mathrm{RSCU}$ in ORF1 & $\mathrm{RSCU}$ in ORF2 \\
\hline \multirow[t]{4}{*}{ Ala } & GCA & 1.238761 & 0.877698 & Leu & CUA & 0.582651 & 0.410596 \\
\hline & GCC & 1.224431 & 1.165468 & & CUC & 1.349825 & 0.397351 \\
\hline & GCG & 0.567437 & 0.014388 & & CUG & 1.188367 & 0.900662 \\
\hline & GCU & 0.969371 & 1.942446 & & CUU & 1.107137 & 0.821192 \\
\hline \multirow[t]{6}{*}{ Arg } & AGA & 1.266604 & 1.481013 & & UUA & 0.498412 & 1.350993 \\
\hline & AGG & 2.026193 & 3.341772 & & UUG & 1.273609 & 2.119205 \\
\hline & CGA & 0.303087 & 0 & Lys & AAA & 0.699282 & 0.837209 \\
\hline & CGC & 0.991581 & 1.177215 & & AAG & 1.300718 & 1.162791 \\
\hline & CGG & 0.445276 & 0 & Phe & UUC & 0.909962 & 0.360902 \\
\hline & CGU & 0.967259 & 0 & & UUU & 1.090038 & 1.639098 \\
\hline \multirow[t]{2}{*}{ Asn } & $\mathrm{AAC}$ & 1.562517 & 0.140845 & Pro & CCA & 1.370342 & 2 \\
\hline & AAU & 0.437483 & 1.859155 & & $\mathrm{CCC}$ & 1.204832 & 0.451613 \\
\hline \multirow[t]{2}{*}{ Asp } & GAC & 1.576108 & 0.909091 & & CCG & 0.45541 & 0 \\
\hline & GAU & 0.423892 & 1.090909 & & $\mathrm{CCU}$ & 0.969417 & 1.548387 \\
\hline \multirow[t]{2}{*}{ Cys } & UGC & 1.034803 & 0 & Ser & AGC & 0.969041 & 1.567416 \\
\hline & UGU & 0.965197 & 0 & & AGU & 1.104135 & 3.370787 \\
\hline \multirow[t]{2}{*}{ Gln } & CAA & 0.798416 & 1.651613 & & UCA & 1.437974 & 0 \\
\hline & CAG & 1.201584 & 0.348387 & & UCC & 1.226239 & 0.522472 \\
\hline \multirow[t]{2}{*}{ Glu } & GAA & 0.843523 & 0.8 & & UCG & 0.558562 & 0 \\
\hline & GAG & 1.156477 & 1.2 & & UCU & 0.704048 & 0.539326 \\
\hline \multirow[t]{4}{*}{ Gly } & GGA & 0.669081 & 0.797508 & He & $A \cup A$ & 0.574538 & 0 \\
\hline & GGC & 1.262976 & 0.984424 & & $A \cup C$ & 1.247451 & 0.525 \\
\hline & GGG & 0.944991 & 0.398754 & & AUU & 1.17801 & 2.475 \\
\hline & GGU & 1.122952 & 1.819315 & Tyr & UAC & 1.285714 & 0.086022 \\
\hline \multirow[t]{2}{*}{ His } & CAC & 1.412429 & 0 & & UAU & 0.714286 & 1.913978 \\
\hline & CAU & 0.587571 & 2 & Val & GUA & 0.316211 & 0.763077 \\
\hline \multirow[t]{4}{*}{ Thr } & ACA & 1.212516 & 0.129032 & & GUC & 1.050408 & 0.258462 \\
\hline & ACC & 1.379635 & 2 & & GUG & 1.163066 & 0.615385 \\
\hline & ACG & 0.496292 & 0 & & GUU & 1.470315 & 2.363077 \\
\hline & $\mathrm{ACU}$ & 0.911557 & 1.870968 & & & & \\
\hline
\end{tabular}

positive value and the other one (marked as Group 2) is negative value. Interestingly, all of those strains isolated before 2000 belonged to Group 2.

\subsection{Correlation analysis}

To estimate whether the evolution of RHDV genome on codon usage was regulated by mutation pressure or natural selection, the $\mathrm{A} \%, \mathrm{U} \%, \mathrm{C} \%, \mathrm{G} \%$ and $(\mathrm{C}+\mathrm{G}) \%$ were compared with $A_{3} \%, U_{3} \%, C_{3} \%, G=$ and $\left(C_{3}\right.$ $\left.+G_{3}\right) \%$, respectively (Table 5 ). There is a complex correlation among nucleotide compositions. In detail, $\mathrm{A}_{3} \%, \mathrm{U}_{3} \%, \mathrm{C}_{3} \%$ and $\mathrm{G}_{3} \%$ have a significant negative correlation with $\mathrm{G} \%, \mathrm{C} \%, \mathrm{U} \%$ and $\mathrm{A} \%$ and positive correlation with $\mathrm{A} \%, \mathrm{U} \%, \mathrm{C} \%$ and $\mathrm{G} \%$, respectively. It suggests that nucleotide constraint may influence synonymous codon usage patterns. However, $\mathrm{A}_{3} \%$ has non-correlation with $\mathrm{U} \%$ and $\mathrm{C} \%$, and $\mathrm{U}_{3} \%$ has noncorrelation with $\mathrm{A} \%$ and $\mathrm{G} \%$, respectively, which haven't indicated any peculiarity about synonymous codon usage. Furthermore, $\mathrm{C}_{3} \%$ and $\mathrm{G}_{3} \%$ have non- correlation with $\mathrm{A} \%, \mathrm{G} \%$ and $\mathrm{U} \%, \mathrm{C} \%$, respectively, indicating these data don't reflect the true feature of synonymous codon usage as well. Therefore, linear regression analysis was implemented to analyze the correlation between synonymous codon usage bias and nucleotide compositions. Details of correlation analysis between the first two principle axes $\left(f_{1}\right.$ and $\left.f_{2}\right)$ of each RHDV genome in COA and nucleotide contents were listed in Table 6 . In surprise, only $\mathrm{f} 2$ values are closely related to base nucleotide $A$ and $G$ content on the third codon position only, suggesting that nucleotide $\mathrm{A}$ and $\mathrm{G}$ is a factor influencing the synonymous codon usage pattern of RHDV genome. However, $f_{1}$ value has non-correlation with base nucleotide contents on the third codon position; it is observably suggest that codon usage patterns in RHDV were probably influenced by other factors, such as the second structure of viral genome and limits of host. In spite of that, compositional constraint is a factor shaping the pattern of synonymous codon usage in RHDV genome. 


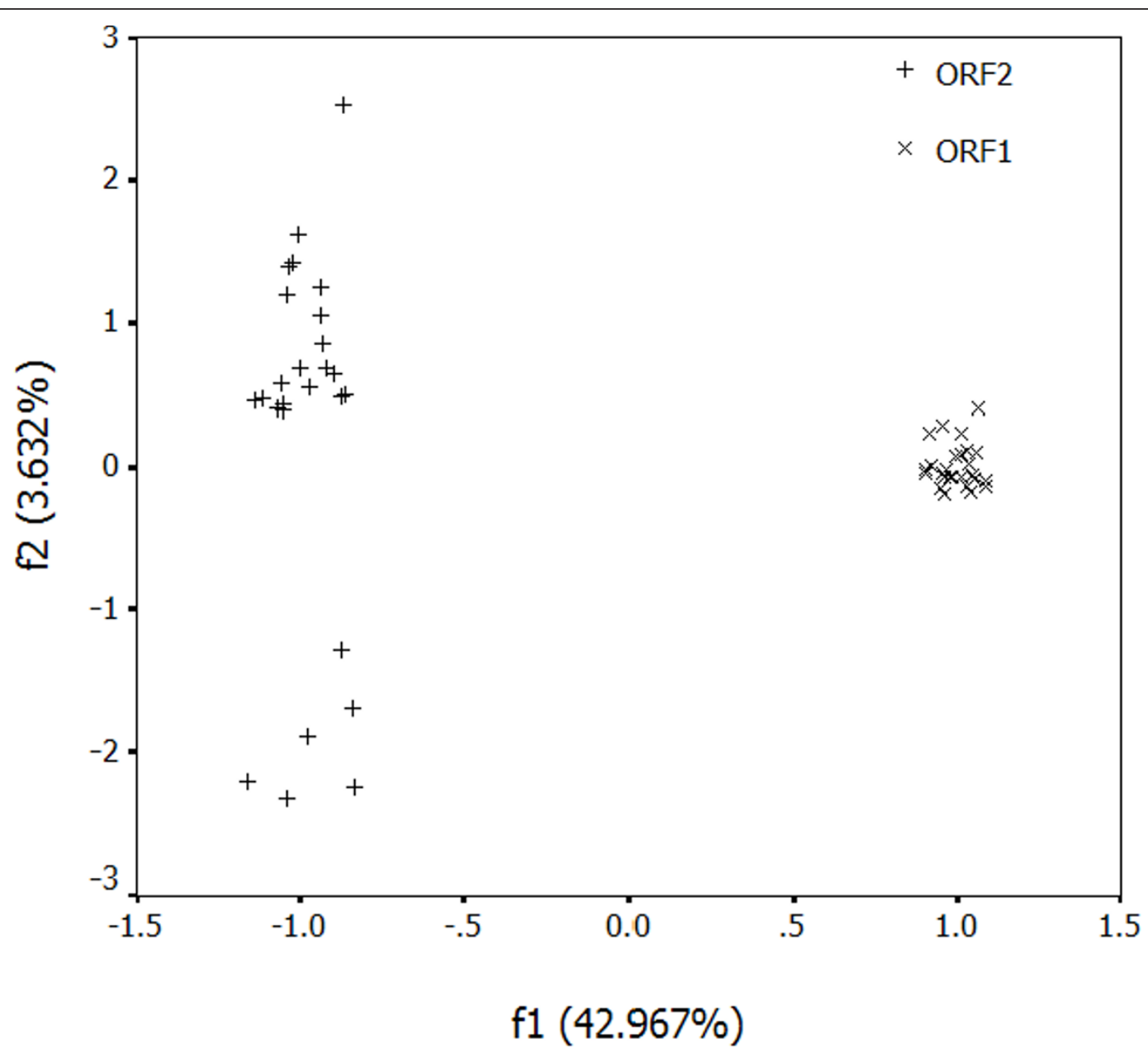

The first axis $\left(f_{1}^{\prime}\right)$ accounts for $42.967 \%$ of the total variation, and the second axis $\left(f^{\prime}\right)$ accounts for $3.632 \%$ of the total variation.

Figure 1 A plot of value of the first and second axis of RHDV genome in COA. The first axis $\left(f_{1}\right)$ accounts for $42.967 \%$ of the total variation, and the second axis $\left(f_{2}\right)$ accounts for $3.632 \%$ of the total variation.

Table 5 Summary of correlation analysis between the $A$, $U, C$, G contents and $A_{3}, U_{3}, C_{3}, G_{3}$ contents in all selected samples

\begin{tabular}{|c|c|c|c|c|c|}
\hline & $A_{3} \%$ & $\mathrm{U}_{3} \%$ & $\mathrm{C}_{3} \%$ & $\mathrm{G}_{3} \%$ & $\left(C_{3}+G_{3}\right) \%$ \\
\hline$A \%$ & $r=0.869^{* *}$ & $\begin{array}{c}r= \\
-0.340^{N S}\end{array}$ & $\begin{array}{c}r= \\
-0.358^{\text {NS }}\end{array}$ & $\begin{array}{c}r= \\
-0.865^{* *}\end{array}$ & $\begin{array}{c}r= \\
-0.266^{* *}\end{array}$ \\
\hline U\% & $r=-0.436^{\mathrm{NS}}$ & $r=0.921^{* *}$ & $\begin{array}{c}r= \\
-0.902^{* *}\end{array}$ & $\begin{array}{c}r= \\
-0.366^{\text {NS }}\end{array}$ & $\begin{array}{c}r= \\
-0.652^{* *}\end{array}$ \\
\hline $\mathrm{C} \%$ & $r=0.376^{\mathrm{NS}}$ & $\begin{array}{c}r= \\
-0.919^{* *}\end{array}$ & $r=0.932^{* *}$ & $\begin{array}{c}r= \\
-0.352^{\text {NS }}\end{array}$ & $r=0.692^{* *}$ \\
\hline G\% & $r=-0.860^{* *}$ & $\begin{array}{c}r= \\
-0.377^{\text {NS }}\end{array}$ & $\begin{array}{c}r= \\
-0.437^{N S}\end{array}$ & $r=0.910^{* *}$ & $r=0.220^{* *}$ \\
\hline $\begin{array}{c}(\mathrm{C} \\
+\mathrm{G}) \%\end{array}$ & $r=\underset{N S}{-0.331}$ & $\begin{array}{c}r= \\
-0.649^{* *}\end{array}$ & $r=0.636^{* *}$ & $r=0.399^{*}$ & $r=0.915^{* *}$ \\
\hline
\end{tabular}

${ }^{a} r$ value in this table is calculated in each correlation analysis.

NS means non-significant $(p>0.05)$.

* means $0.01<p<0.05$

${ }^{* *}$ means $p<0.01$
Table 6 Summary of correlation analysis between the f1, f2 contents and $A_{3}, U_{3}, C_{3}, G_{3}, C_{3}+G_{3}$ contents in all selected samples

\begin{tabular}{cll}
\hline Base compositions & $\boldsymbol{f}_{\boldsymbol{1}^{\prime}}(\mathbf{4 2 . 9 6 7 \% )}$ & $\boldsymbol{f}_{\mathbf{2}}{ }^{\prime}(\mathbf{3 . 6 3 2} \%)$ \\
\hline $\mathrm{A}_{3} \%$ & $r=-0.051^{\mathrm{NS}}$ & $r=-0.740^{* *}$ \\
$U_{3} \%$ & $r=0.243^{\mathrm{NS}}$ & $r=0.314^{\mathrm{NS}}$ \\
$C_{3} \%$ & $r=-0.291^{\mathrm{NS}}$ & $r=-0.298^{\mathrm{NS}}$ \\
$\mathrm{G}_{3} \%$ & $r=0.108^{\mathrm{NS}}$ & $r=0.723^{* *}$ \\
$\left(\mathrm{C}_{3}+\mathrm{G}_{3}\right) \%$ & $r=-0.216^{\mathrm{NS}}$ & $r=0.205^{\mathrm{NS}}$ \\
\hline
\end{tabular}

${ }^{a} \mathrm{r}$ value in this table is calculated in each correlation analysis. NS means non-significant.

* means $0.01<\mathrm{p}<0.05$

**means $p<0.01$ 


\section{Discussion}

There have been more and more features that are unique to RHDV within the family Caliciviridae, including its single host tropism, its genome and its VP10 as a structural protein with unknown function. After we analyzed synonymous codon usage in RHDV (Table 2), we obtained several conclusions and conjectures as followed.

\subsection{Mutational bias as a main factor leading to synonymous codon usage variation}

ENC-plot, as a general strategy, was utilized to investigate patterns of synonymous codon usage. The ENC-plots of ORFs constrained only by a $\mathrm{C}_{3}+\mathrm{G}_{3}$ composition will lie on or just below the curve of the predicted values [18]. ENC values of RHDV genomes were plotted against its corresponding $\left(\mathrm{C}_{3}+\mathrm{G}_{3}\right) \%$. All of the spots lie below the curve of the predicted values, as shown in Figure 2, suggesting that the codon usage bias in all these 30 RHDV genomes is principally influenced by the mutational bias.
4.2 A proof for codon usage bias as a factor reducing the expression of VP10

As we know, the efficiency of gene expression is influenced by regulator sequences or elements and codon usage bias. It reported that the RNA sequence of the 3terminal 84 nucleotides of ORF1were found to be crucial for VP10 expression instead of the encoded peptide. VP10 coding by ORF2 has been reported as a low expressive structural protein against VP60 coding by ORF1 [5]. And its efficiency of translation is only $20 \%$ of VP60. According to results showed by Table 4, it revealed the differences in codon usage patterns of two ORFs, which is a possible factor reducing the expression of VP10.

\subsection{Negative selective constraints on the RHDV whole genome}

Although VP10 encoded by ORF2, as a minor structural protein with unknown functions, has been described by $\mathrm{LIU}$ as a nonessential protein for virus infectivity, the $\omega$

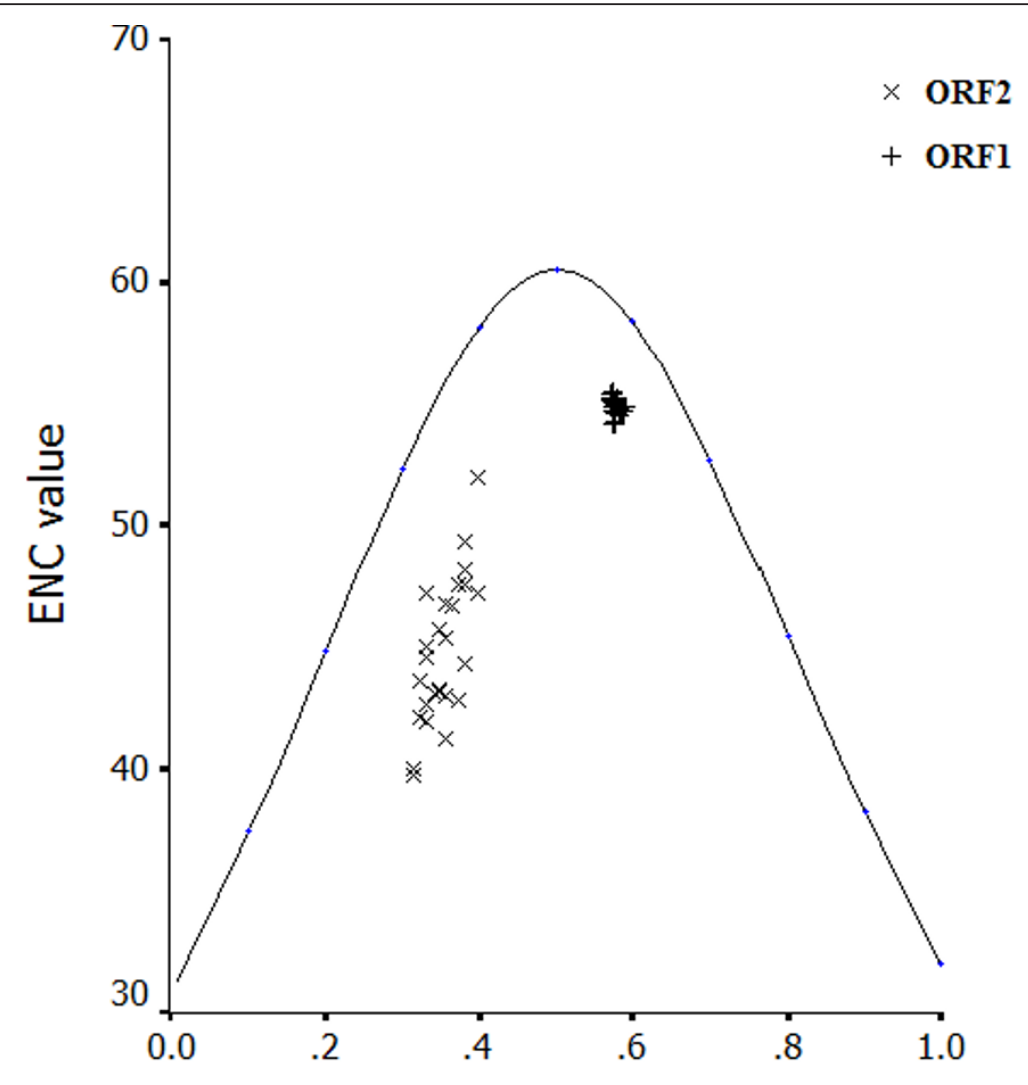

\begin{abstract}
GC3\%
The continuous curve plots the relationship between $\mathrm{GC} 3 \mathrm{~s}$ and $\mathrm{ENC}$ in the absence of selection. All of spots lie below the expected curve.
\end{abstract}

Figure 2 Effective number of codons used in each ORF plotted against the GC3s. The continuous curve plots the relationship between GC3s and ENC in the absence of selection. All of spots lie below the expected curve. 
Table 7 Summary of correlation analysis between ENC value of ORF1 and ENC value of ORF2

\begin{tabular}{ccc}
\hline & ENC value of ORF1 & ENC value of ORF2 \\
\hline ENC value of & $r=1, p=0$ & $r=0.230, p=0.222>$ \\
ORF1 & & 0.05 \\
ENC value of & $r=0.230, p=0.222>$ & $r=1, p=0$ \\
ORF2 & 0.05 & \\
\hline
\end{tabular}

value of ORF2 suggests VP10 plays an important role in the certain stage of whole RHDV lifecycle. After combining with low expression and $\omega$ value of VP10, we conjectured that VP10 might be beneficial for the replication, release or both of virus by inducing infected cell apoptosis initiate by RHDV. This mechanism has been confirmed in various positive-chain RNA viruses, including coxsackievirus, dengue virus, equine arterivirus, footand-mouth disease virus, hepatitis $\mathrm{C}$ virus, poliovirus, rhinovirus, and severe acute respiratory syndrome [23-29], although the details remain elusive.

\subsection{Independent evolution of ORF1 and ORF2}

As preceding description, ENC reflects the evolution of codon usage variation and nucleotide composition to some degree. After the correlation analysis of ENC values between ORF1 and ORF2 (Table 7), the related coefficient of ENC values of two ORFs is 0.230 , and $p$ value is 0.222 more than 0.05 . These data revealed that no correlation existed in ENC values of two ORFs, indicating that codon usage patterns and evolution of two ORFs are separated each other. Further, this information maybe helps us well understand why RSCU and ENC between two ORFs are quite different.

\subsection{A possible genotyping basis}

Interestingly, we found that relatively isolated spots from ORF2 tend to cluster into two groups: the ordinate value of one group (marked as Group 1) is positive value and the other one (marked as Group 2) is negative value. And all of those strains isolated before 2000 belonged to Group 2, including Italy-90, RHDV-V351, RHDV-FRG, BS89, RHDV-SD and M67473.1. Although RHDV has been reported as only one type, this may be a reference on dividing into two genotypes.

\section{Conclusion}

In this report, we firstly analyzed its genome and two open reading frameworks (ORFs) from this aspect of codon usage bias. Our researches indicated that mutation pressure rather than natural is the most important determinant in RHDV with high codon bias, and the codon usage bias is nearly contrary between ORF1 and ORF2, which is maybe one of factors regulating the expression of VP60 (encoding by ORF1) and VP10 (encoding by ORF2). Furthermore, negative selective constraints on the RHDV whole genome implied that VP10 played an important role in RHDV lifecycle. We conjectured that VP10 might be beneficial for the replication, release or both of virus by inducing infected cell apoptosis initiate by RHDV. According to the results of the principal component analysis for ORF2 of RSCU, we firstly separated 30 RHDV into two genotypes, and the ENC values indicated ORF1 and ORF2 were independent among the evolution of RHDV. All the results will guide the next researches on the RHDV as a reference.

\section{Acknowledgements \\ This work was supported by the fund of Special Social Commonweal Research Programs for Research Institutions (2005DIB4J041, China).}

\section{Authors' contributions}

$\mathrm{XTT}$ and BYL contributed equally to the original draft of the manuscript, and approved the final version. ZL and WQJ contributed to conception and design of the manuscript, and revised the manuscript. LJX is the corresponding author. All authors have read and approved the final manuscript.

\section{Competing interests}

The authors declare that they have no competing interests.

Received: 14 September 2011 Accepted: 1 November 2011 Published: 1 November 2011

\section{References}

1. Karlin S, Mrazek J: What drives codon choices in human genes? J Mol Biol 1996, 262(4):459-472.

2. Sharp PM, Tuohy TM, Mosurski KR: Codon usage in yeast: cluster analysis clearly differentiates highly and lowly expressed genes. Nucleic Acids Res 1986, 14(13):5125-5143.

3. Lesnik T, Solomovici J, Deana A, Ehrlich R, Reiss C: Ribosome traffic in Ecoli and regulation of gene expression. Journal of Theoretical Biology 2000, 202(2):175-185.

4. Shackelton LA, Parrish CR, Holmes EC: Evolutionary basis of codon usage and nucleotide composition bias in vertebrate DNA viruses. J Mol Evol 2006, 62(5):551-563.

5. Mooers $\mathrm{AO}$, Holmes $\mathrm{EC}$ : The evolution of base composition and phylogenetic inference. Trends Ecol Evol 2000, 15(9):365-369.

6. Jenkins GM, Holmes EC: The extent of codon usage bias in human RNA viruses and its evolutionary origin. Virus Res 2003, 92(1):1-7.

7. Zhou J, Liu WJ, Peng SW, Sun XY, Frazer I: Papillomavirus capsid protein expression level depends on the match between codon usage and tRNA availability. J Virol 1999, 73(6):4972-4982.

8. Zhao KN, Liu WJ, Frazer $\mathrm{H}$ : Codon usage bias and $\mathrm{A}+\mathrm{T}$ content variation in human papillomavirus genomes. Virus Res 2003, 98(2):95-104.

9. Karlin S, Blaisdell BE, Schachtel GA: Contrasts in codon usage of latent versus productive genes of Epstein-Barr virus: data and hypotheses. J Virol 1990, 64(9):4264-4273.

10. Sewatanon J, Srichatrapimuk S, Auewarakul P: Compositional bias and size of genomes of human DNA viruses. Intervirology 2007, 50(2):123-132.

11. Van Hemert FJ, Berkhout B, Lukashov W: Host-related nucleotide composition and codon usage as driving forces in the recent evolution of the Astroviridae. Virology 2007, 361(2):447-454.

12. Wirblich C, Thiel HJ, Meyers G: Genetic map of the calicivirus rabbit hemorrhagic disease virus as deduced from in vitro translation studies. $J$ Virol 1996, 70(11):7974-7983.

13. Wirblich C, Sibilia M, Boniotti MB, Rossi C, Thiel HJ, Meyers G: 3C-like protease of rabbit hemorrhagic disease virus: identification of cleavage sites in the ORF1 polyprotein and analysis of cleavage specificity. J Virol 1995, 69(11):7159-7168 
14. Meyers G, Wirblich C, Thiel HJ, Thumfart JO: Rabbit hemorrhagic disease virus: genome organization and polyprotein processing of a calicivirus studied after transient expression of cDNA constructs. Virology 2000, 276(2):349-363.

15. Meyers G: Translation of the minor capsid protein of a calicivirus is initiated by a novel termination-dependent reinitiation mechanism. $J$ Biol Chem 2003, 278(36):34051-34060.

16. Comeron JM, Aguade M: An evaluation of measures of synonymous codon usage bias. J Mol Evol 1998, 47:268-274.

17. Wright F: The "effective number of codons" used in a gene. Gene 1990, 87:23-29.

18. Lu H, Zhao WM, Zheng Y, Wang H, Qi M, Yu XP: Analysis of Synonymous Codon Usage Bias in Chlamydia. Acta Biochim Biophys Sin 2005, 37:1-10.

19. Nei M, Gojobori T: Simple methods for estimating the numbers of synonymous and nonsynonymous nucleotide substitutions. Molecular Biology and Evolution 1986, 3:418-426.

20. Tamura K, Dudley J, Nei M, Kumar S: MEGA4: Molecular Evolutionary Genetics Analysis (MEGA) software version 4.0. Molecular Biology and Evolution 2007, 24:1596-1599.

21. Mardia KV, Kent JT, Bibby JM: Multivariate analysis NewYork. Academic press; 1979.

22. Ewens WJ, Grant GR: Statistical Methods in Bioinformatics NewYork. Springer; 2001.

23. Egger D, Wolk B, Gosert R, Bianchi L, Blum HE, Moradpour D, Bienz K: Expression of Hepatitis $C$ virus proteins induced distinct membrane alterations including a candidate viral replication complex. J Virol 2002, 76(12):5974-5984.

24. Espert L, Codogno P, Biard-Piechaczyk K: Involvement of autophagy in viral infections: antiviral function and subversion by viruses. J Mol Med 2007, 85:811-823.

25. Jackson WT, Giddings TH, Taylor MP, Mulinyawe S, Rabinovitch M, Ropito R, Kirkegaard K: Subversion of cellular autophagosomal machinery by RNA viruses. PLOS Biol 2005, 3(5):e156.

26. Lee YR, Lei HY, Liu MT, Wang JR, Chen SH, Jiang-Shieh YF, Lin YS, Yeh TM, Liu CC, Liu HS: Autophagic machinery activated by dengue virus enhances virus replication. Virology 2008, 374:240-248

27. Pedersen KW, van der Meer $Y$, Roos N, Snijder EJ: Open reading frame 1aencoded subunits of the arterivirus replicase induce endoplasmic reticulum derived double-membrane vesicles which carry the viral replication complex. J Virol 1999, 73(3):2016-2026.

28. Wileman T: Aggresomes and autophagy generate sites for virus replication. Science 2006, 312:875-878.

29. Wong J, Zhang J, Xiaoning S, Gao G, Mao I, McManus BM, Luo H: Autophagosome supports Coxsackievirus B3 replication in host cells. J Virol 2008, 82(18):9143-9153.

doi:10.1186/1743-422X-8-494

Cite this article as: Tian et al: Bioinformatics analysis of rabbit

haemorrhagic disease virus genome. Virology Journal 2011 8:494. 